\title{
Entgrenzung in Industrie und Altenpflege: Perspektiven erweiterter Handlungsfähigkeit der Beschäftigten
}

\author{
Unzureichende Sicherheiten und Überlastungssituationen, die in entgrenzten Arbeits- \\ und Lebenssituationen auftreten, werden von den Beschäftigten überwiegend individuell \\ bewältigt. Die gesellschaftlichen Strukturen, die zu diesen Unsicherheiten führen, bleiben \\ dadurch unverändert oder werden sogar stabilisiert. In einer Untersuchung der unter- \\ schiedlichen Arbeits- und Lebensbedingungen von Erwerbstätigen in Altenpflegeheimen \\ und Industrieunternehmen gehen wir empirisch der Frage nach, auf welche subjektiven \\ Begründungszusammenhänge sich individualisiertes Handeln im Alltagsdenken stützt. \\ Gleichzeitig untersuchen wir, wo sich darin Brüche und unbearbeitete Widersprüche fin- \\ den, die Ausgangspunkte von (Selbst-)Verständigungsprozessen und der Entwicklung \\ kollektiver Handlungsstrategien sein können.
}

IRIS NOWAK, JETTE HAUSOTTER, GABRIELE WINKER

\section{Individualisiertes Handeln als erklärenswertes Phänomen}

Der Begriff Entgrenzung bezeichnet Prozesse, in denen fordistische Normalitätsstandards in der Erwerbsarbeit an Bedeutung verlieren. Für die individuelle Lebensgestaltung eröffnet dies teilweise neue Freiräume. Allerdings findet Entgrenzung unter Bedingungen statt, die darauf zielen, immer größere Bereiche des menschlichen Lebens an Marktprinzipien auszurichten. Infolge dieser Vermarktlichung geht Entgrenzung für viele Menschen mit Überlastungen und neuen Einschränkungen einher. Die Zahl prekärer Beschäftigungsverhältnisse wächst. Aber auch Unsicherheiten innerhalb jener Erwerbsarbeitsverhältnisse, die relativ sicher, gut bezahlt und sozial abgesichert sind, nehmen zu. ${ }^{\bullet}$ Erweiterte Analysen von Prekarisierung beziehen zudem den Lebenszusammenhang ein (vgl. WSIMitteilungen 8/2011). Damit werden verstärkt Planungsund Gestaltungsunsicherheiten sowie Verwundbarkeiten thematisiert, die sich durch die „mangelnde Passfähigkeit" (Klenner/Pfahl 2011, S. 417) unterschiedlicher Lebensbereiche, wie das Erwerbsleben, die Sorge für andere und die eigene Regeneration, ergeben. So kann die Gestaltung des eigenen Lebens allein dadurch unsicher sein, dass private Fürsorgenetzwerke brüchig werden, die Gesundheit leidet oder sozialstaatliche Leistungen im Bedarfsfall schwer zugänglich bzw. unzureichend sind.
Das Forschungsprojekt „Handlungsfähigkeit in entgrenzten Verhältnissen“" ${ }^{20}$ setzt an der Tatsache an, dass diese abnehmenden Sicherheiten und Planbarkeiten von den Betroffenen überwiegend individuell verarbeitet werden. Wir sehen diese vorherrschende Individualisierung als Phänomen an, das der genaueren Betrachtung bedarf, da Planungsunsicherheit und soziale Verwundbarkeit einem gesellschaftlichen Widerspruch „zwischen betrieblichen Verwertungs- und individuellen Reproduktionsinteressen als Arbeitskraft“ (Jürgens 2006, S. 201) entspringen, der sich durch individuelle Bewältigungsstrategien nicht aufheben lässt. Indem Menschen die Bedingungen, unter denen sie handeln (müssen), aktiv mit Bedeutungen versehen, positionieren sie sich innerhalb der Konflikte über die Regulierung dieses Widerspruchs. Sie tragen damit zu bestimmten gesellschaftlichen Entwicklungen bei, während sie andere

\footnotetext{
Zu nennen sind hier der permanente Wandel von Arbeitsinhalten und -formen bei verknappten Ressourcen und rigiden Zielvorgaben, hohe Anforderungen an raum-zeitliche Flexibilität, sinkende Reallöhne und die disziplinierende Wirkung prekärer Beschäftigung auf die Normalarbeitenden. Zu den Folgen gehören fehlende Sinnstiftung, physischer und psychischer Verschleiß und Arbeitskrisen (Jürgens 2011, S. 380f.; Lehndorff 2003, S. 155, 2005, S. 206ff.).
}

2 Das Projekt wurde von den Autorinnen dieses Beitrages (Leitung Gabriele Winker) von 2009 bis 2011 an derTU Hamburg-Harburg durchgeführt, vgl. Nowak et al. (2012). 
verhindern. Die relativ reibungslose Durchsetzung struktureller Transformationsprozesse wie Vermarktlichung und Entgrenzung setzt voraus, dass Menschen sich aktiv in die neuen Verhältnisse einpassen. Durch rein individualisierte Bewältigungsweisen entsprechen sie dem neuen gesellschaftlichen Arrangement, das sie zur „Eigenaktivität im Interesse der gesellschaftlichen Gemeinschaft anhält“ (Lessenich 2008, S. 83), ihnen also nahelegt, durch private Vorsorge, Eigeninitiative, Prävention und andere „Varianten der Aktivierung von Eigenverantwortung“ (ebd.) ein produktives Mitglied der Gesellschaft zu sein.

Um langfristig sicher über das eigene Leben bestimmen zu können, müssen Menschen in die Veränderung gesellschaftlicher Strukturen eingreifen. Wir sehen es als Voraussetzung für solidarisch-kollektive Handlungsformen an, dass Menschen sich der Grenzen ihrer individualisierten Strategien bewusst werden. Dafür ist es wichtig, die subjektiven Gründe für diese Strategien nachzuvollziehen und deren Brüche und Widersprüche zu begreifen. Um einen Beitrag zu (Selbst-)Reflexionsprozessen darüber zu leisten, wo Ansatzpunkte für politische Initiativen in und außerhalb von Betrieben sein können (z.B. von Betriebsräten, Gleichstellungsbeauftragten oder Elterninitiativen), haben wir empirisch untersucht, wie Erwerbstätige die Belastungen, denen sie durch die Entgrenzung ihrer Arbeits- und Lebensverhältnisse ausgesetzt sind, beschreiben, welche Handlungsmöglichkeiten sie sehen und wie sie diese begründen.

\section{Entgrenzung und Vermarktlichung als Handlungsbedingungen}

In der Arbeitssoziologie werden Entgrenzung und Vermarktlichung von Erwerbsarbeit als ambivalente Angelegenheiten diskutiert. Einerseits können entsprechende Steuerungsformen den Beschäftigten mehr Entscheidungskompetenzen und neue Möglichkeiten geben, subjektive Zielsetzungen in der Erwerbsarbeit zu verwirklichen (vgl. z.B. Lohr/Nickel 2005). Zugleich werden durch vermarktlichte Unternehmensstrukturen verschärfte Leistungsansprüche bei knapperen Ressourcen an die Beschäftigten herangetragen. Beschäftigte „erleben,empowerment ‘ als selbst gemanagte Intensivierung von Arbeit" (Lehndorff 2003, S. 165) und bezahlen dies oft mit einem „Verlust an Lebensqualität“" (Kratzer 2003, S. 202). Vor diesem Hintergrund arbeiten Analysen der Handlungsmöglichkeiten von Beschäftigten nicht länger mit Dichotomien und einfachen Gegenüberstellungen z.B. von wachsender Freiheit und zunehmendem Zwang (ebd., S. 15). Vielmehr geht es um das Verstehen von Gleichzeitigkeiten und Widersprüchen zwischen Entlastungen und Belastungen und zwischen „,eigensinniger 'Subjektivität“ und ihrer fremdbestimmten Nutzung (ebd. S. 16). Die „Gleichzeitigkeit von erweiterter
Teilhabe und neuer sozialer Verwundbarkeit“ beinhaltet „riskante Chancen“ für die „Demokratisierung“ und „nachhaltige Entwicklung von Arbeit“ (Lohr/Nickel 2005, S. 209).

Vermarktlichte Steuerungsformen setzen bewusst auf den Abbau hierarchischer Kommandosysteme (Glißmann 2000). Die Fremdbestimmung von Handeln setzt sich vermittelt „über ihr eigenes Gegenteil, nämlich die Selbstbestimmung oder Autonomie der Individuen“ durch (Peters/ Sauer 2005, S. 24). Die steigenden Leistungsanforderungen treten den Beschäftigten als Ausdruck von abstrakten Marktmechanismen gegenüber, d.h. als sachliche Zwänge, mit denen sie selbstverantwortlich umgehen müssen. Forderungen nach guten Arbeitsbedingungen an die Unternehmen oder Beschäftigtenvertretungen scheinen in Anbetracht dieser Erfahrung sinnlos. Entsprechend werden Konsequenzen für die individuelle Reproduktion (Fragen nach Gesundheit, Arbeitszeit etc.) oft nicht zum Gegenstand kollektiver Aushandlungen und Begrenzungen gemacht, sondern innerhalb des selbst gestalteten Arbeitens individuell bewältigt. Will man darüber hinauskommen, ist die Frage, wie an die erweiterten Verantwortungsspielräume der subjektivierten Arbeitsweisen angeschlossen werden kann, um zugleich eine „offensive Partizipationspolitik“ (Dörre 2002, S. 74) durchzusetzen, die es allen Beschäftigten ermöglicht, in die Bedingungen, unter denen sie arbeiten, gestaltend einzugreifen. Dafür braucht es Angebote zur Unterstützung der individuellen Formen der Interessenvertretung, die gleichzeitig Anschlüsse an eine „solidarische Arbeitspolitik“ (Lohr/Nickel 2005, S. 224) erlauben. Und es braucht eine erneuerte „Politisierung des Arbeitszeitkonflikts im Betrieb“ (Lehndorff 2005, S. 216).

Diese für unsere Forschung zentralen arbeitssoziologischen Erkenntnisse haben wir in zwei Hinsichten erweitert, die wir im Folgenden kurz begründen: Erstens vermeiden wir eine erwerbszentrierte Perspektive auf Arbeit, indem wir den Lebenszusammenhang in unsere Analyse einbeziehen. Zweitens hinterfragten wir die Dominanz industrieller Erwerbsarbeit, die in den Untersuchungen und Zeitdiagnosen der Arbeitsforschung vorherrscht (Lohr/Nickel 2005, S. 215). Unter diesen Prämissen gilt unser Augenmerk Beschäftigten im Industrie- und im Pflegebereich.

\subsection{Zugespitzte Privatförmigkeit von Reproduktion}

Das Handbuch Arbeitssoziologie (Voß et al. 2010) zeigt, dass dezidiert geschlechterkritische Perspektiven sowie Fragen nach dem Lebenszusammenhang Eingang in den Mainstream der Forschungsthemen gefunden haben. Aber gleichzeitig bestimmen gerade in der Analyse aktueller Rationalisierungsformen nach wie vor betriebliche bzw. unternehmensgesteuerte Prozesse den Blick der Disziplin. Dabei sind neue Verwertungsformen von Arbeitskraft nicht $\mathrm{zu}$ verstehen, wenn unberücksichtigt bleibt, wie reproduktive Bedürfnisse befriedigt werden und wie diese zu Anforderungen aus der Erwerbsarbeit ins Verhältnis gesetzt 
werden. Die Durchsetzung neuer Rationalisierungsformen setzt voraus, dass Menschen eine Lebensweise entwickeln, die mit den subjektivierten und entgrenzten Anforderungen korrespondiert und zugleich einen Ausgleich hierzu schafft (Nowak 2007). Winker/Carstensen (2007) haben in feministischer Erweiterung der Arbeitskraftunternehmerthese von Voß/Pongratz (1998) beschrieben, dass die Handlungsprämissen des Arbeitskraftunternehmers (Selbstkontrolle, Selbstrationalisierung und Selbstökonomisierung) gleichfalls Anforderungen an die Fürsorgearbeit und das Reproduktionshandeln stellen. Sie sprechen sich für eine Perspektive aus, welche die ,aktive Koordinierung und Synchronisation von Tätigkeiten ganz verschiedener Art in allen Lebensbereichen betont" (Winker/Carstensen 2007, S. 282). Mit ihrem Konzept der Arbeitskraftmanagerin/des Arbeitskraftmanagers möchten sie „die Reproduktionsarbeit analytisch einbeziehen, die sich - ähnlich wie die Erwerbsarbeit - flexibilisiert, verdichtet und von den Individuen eigenständig zu organisieren ist" (ebd., S. 278). Jurczyk et al. (2009) beschäftigen sich vom gleichen Ausgangspunkt aus empirisch mit dem Handeln von Menschen in entgrenzten Arbeits- und Lebensbedingungen. Sie zeigen, dass die Anforderungen an ,individuelles Grenzmanagement“ (ebd., S. 61, 313ff.) im Familienkontext aufgrund der notwendigen Koordinierungsleistungen besonders hoch sind. Dies gelte auch für die alltägliche Gestaltung des Verhältnisses von Familien- und Erwerbssphäre. Im Zuge der „Entgrenzung von Geschlechterverhältnissen“ (ebd., S. 113), welche aus Sicht des Autorenteams die Schnittstelle von betrieblicher und familiärer Sphäre darstellen, wird die Kooperation zwischen Menschen vermehrt durch Aushandlung, Konflikt und eine Aufkündigung etablierter Kooperationsformen geprägt. Jurczyk et al. zeigen vielfältige Formen auf, in denen Menschen Entgrenzungen aktiv gestalten und neue Begrenzungen vornehmen, die oft ein widerständiges Handeln gegenüber betrieblichen Anforderungen „zur Verteidigung von persönlichen und familialen Interessen“ (ebd., S. 316) einschließen. Da sie in ihrer Studie jedoch das individuelle Grenzmanagement als Forschungsgegenstand konzeptionell voraussetzen, zeigen auch ihre Ergebnisse lediglich individuelle Praxen auf. Wie in der Einleitung dargestellt, betrachteten wir im Unterschied hierzu diese Individualisierung als erklärungswürdiges Phänomen, das - auch durch die Betroffenen selbst - einer Kritik unterzogen werden muss, sofern man verallgemeinerbare Formen finden will, um selbst gesetzte Grenzen langfristig abzusichern.

\subsection{Entgrenzung in Industrieunternehmen und Altenpflege}

Die Frage nach neuen Arbeits- und Partizipationsformen wird in der Arbeitssoziologie vielfach auf den Bereich der Industriearbeit bezogen. Zu den Folgen von Vermarktlichung im Dienstleistungsbereich werden Analogien gezogen, die etwa Lehndorff auf die Formel zuspitzt: „Industriearbeit wird ,tertiarisiert', Dienstleistungsarbeit wird ,industrialisiert" (Lehndorff 2003, S. 161), wobei er sich mit Letzterem auf die Bereiche Einzelhandel, IT-Dienstleistungen und Banken bezieht. Es fehlen aber weitgehend Diskussionen darüber, inwiefern die arbeitssoziologischen Analysen im Bereich sozialer Dienstleistungen zutreffen (vgl. Nowak 2011). Große Unterschiede lassen sich exemplarisch an den unterschiedlichen Ausprägungen von Entgrenzung verdeutlichen, die Ingenieurinnen/Ingenieure und Facharbeiterinnen/Facharbeiter ${ }^{3}$ in Industrieunternehmen sowie Altenpflegekräfte erleben.

Da die Industrie der Kernbereich von Normalarbeit im Fordismus war, sind Entgrenzungsprozesse hier am eindeutigsten als Auflösung fordistischer Regulierungen zu beschreiben. Dies gilt trotz Unterschieden sowohl für Ingenieure als auch für Facharbeiter beiderlei Geschlechts. Vermarktlichung und Entgrenzung sind wesentliche Aspekte von Rationalisierungsstrategien, die mit diversen Mitteln auf die Mobilisierung der Produktionsintelligenz von Beschäftigten setzen. Sowohl im Engineering als auch in der Fertigung gibt es dabei eine Gleichzeitigkeit der Erweiterung der Autonomie der Beschäftigten und Gegentendenzen einer (Re-)Taylorisierung. Darüber hinaus verlieren Tarifverträge an Prägekraft für die realen Arbeitsbedingungen, der Trend geht zu einer Dezentralisierung und Verbetrieblichung der Tarifpolitik. Gewerkschaften verlieren an Durchsetzungsfähigkeit und an Attraktivität als Akteure der Durchsetzung kollektiver Mindeststandards. Die „Krisensymptome“ (Bispinck 2003, S. 403) in den industriellen Beziehungen drücken sich hier auch als nachlassende Organisierungsmacht aus. Gleichzeitig könnten hochqualifizierte Angestellte durch den Verlust ihrer privilegierten Stellung in Unternehmen ein neues „Arbeitnehmerbewusstsein“ entwickeln, das offen für kollektive Formen der Interessendurchsetzung ist (Boes/Kämpf 2010).

In der Altenpflege hingegen war ein fordistisch reguliertes Normalarbeitsverhältnis nie von Bedeutung (Dunkel 2005, S. 242). Bis in die 1960er Jahre hinein wurde diese Tätigkeit in Westdeutschland vor allem in christlichen Einrichtungen geleistet und als Ausdruck der Liebe zu Gott bzw. einer spezifisch weiblichen Veranlagung betrachtet (ebd.; Schweiger 2011, S. 21f.; Kumbruck 2009, S. 12ff.). Da hierbei Grenzen zwischen der Pflegearbeit und dem sonstigen Leben oft nicht vorgesehen waren, sprechen wir von einer traditionellen Entgrenztheit dieser Arbeitsverhältnisse. Erst ab den 1960er Jahren entwickelte sich Altenpflege zu einem „normalen Frauenberuf“ (Kumbruck 2009, S. 29).

Pflegearbeit setzt eine subjektive Involviertheit der Beschäftigten in die Beziehungen zu den Pflegebedürftigen voraus (vgl. Böhle 1999; Kumbruck 2009). Zugleich wird Pflege durch Vermarktlichung, die mit Einführung der Pflegeversicherung 1996 einsetzte, immer mehr vom Leitbild

(3) Facharbeiter bezeichnet in unserem Sample Mechanikerinnen/Mechaniker und Elektronikerinnen/Elektroniker in der Produktion mit einschlägiger Berufsausbildung. 
einer zweckrationalen Organisation und zeitökonomischen Rationalisierung durchdrungen (z.B. Böhle 1999, S. 174, 179f.). Subjektivierung und verstärkte Taylorisierung und Rationalisierung sind darin keine Gegensätze. In der Altenpflege finden wir vielmehr wie auch in anderen Dienstleistungsbranchen einen „subjektivierten Taylorismus“ (Matuschek et al. 2008, S. 50), mit dem Unternehmen die empathischen und kommunikativen Potenziale der Beschäftigten innerhalb enger Rahmenvorgaben optimal zu nutzen versuchen (Kratzer 2003, S. 54). Dass Selbstorganisierungsprozesse und kollektive Interessenvertretung im Bereich Pflege aufgrund ihrer historischen Prägung, aber auch aufgrund ihrer spezifischen Eigenschaft als Tätigkeit, bei der es um die Sorge für Menschen geht, von grundlegend anderen Voraussetzungen als im industriellen Bereich ausgehen müssen, ist bisher kaum untersucht worden (Nowak 2011).

\section{Empirische Befunde}

Unsere Studie (vgl. Fußnote 2) beruht auf 28 narrativen Interviews mit Beschäftigten in Altenpflegeheimen und mit Ingenieurinnen/Ingenieuren und Facharbeiterinnen/Facharbeitern eines Großunternehmens der Metallbranche. Interviewthemen waren Anforderungen und Konflikte im Alltag, besonders im Hinblick auf die Vereinbarung verschiedener Arbeits- und Lebensbereiche, sowie Handlungsstrategien in den thematisierten Konflikten. Wie die Befragten ihre Situation und Handlungsmöglichkeiten wahrnehmen, arbeiteten wir anhand ihrer Subjektkonstruktionen heraus. D.h. wir analysierten, wie die Befragten die gesellschaftlichen Strukturen und ihre eigene Identität beschreiben und auf welche Normen, Alltagsweisheiten etc. sie dabei zurückgreifen. ${ }^{\oplus}$ Mit dieser Analyse der Subjektkonstruktionen wurde die Frage nach der Handlungsfähigkeit der Befragten verknüpft. Hier lehnten wir uns an die Unterscheidung von restriktiver und erweiterter Handlungsfähigkeit an, wie sie die Kritische Psychologie vornimmt (Holzkamp 1983). Als restriktive Handlungsfähigkeit verstehen wir solche Aspekte der Subjektkonstruktionen, in denen Menschen ihre Lebensbedingungen als gegeben hinnehmen und lediglich daran arbeiten, ihr Leben innerhalb dieser Verhältnisse zu gestalten. Als erweiterte Handlungsfähigkeit bezeichnen wir solche Aspekte der Subjektkonstruktionen, in denen Menschen die gesellschaftliche Vermitteltheit der eigenen Existenz erkennen und ein Handeln anstreben, das in diese Verhältnisse gestaltend eingreift. Dabei bezeichnen beide Begriffe nicht einander ausschließende Arten des Handelns, sondern Aspekte von Denk- und Praxisformen, die Menschen aufgrund der widersprüchlichen Eingelassenheit von Subjektivität in die herrschenden Verhältnisse durchaus auch fragmentiert und gleichzeitig entwickeln. Die Frage nach erweiterter Handlungsfähigkeit ist der Standpunkt, von dem aus wir diese Widersprüch- lichkeit diskutieren. Uns interessiert, wie sich die Befragten in ihren Subjektkonstruktionen auf die gesellschaftliche Vermitteltheit ihrer Lebensbedingungen beziehen. In diesem Sinne dient uns Handlungsfähigkeit als „Instrument zur Analyse von gesellschaftlich vermittelten Widersprüchen im Handeln, Denken und Empfinden“ (Markard 2011, S. 125).

Insgesamt fanden wir bei fast allen Befragten eine Prekarisierung des Lebenszusammenhangs. Die konkrete Form und das Ausmaß dieser Prekarität variierten dabei abhängig vom Berufsfeld sowie den Reproduktionsanforderungen der Beschäftigten. Dies fassen wir im folgenden Abschnitt (3.1) zusammen. Anschließend stellen wir die Wahrnehmungsweisen und Bewältigungsstrategien der Befragten entlang von vier Gruppen dar (3.2).

\subsection{Prekärer Lebenszusammenhang durch Entgrenzung}

Die klassische Form von Mehrarbeit als Überstunden fanden wir im Industrie- und im Pflegebereich. Es gibt darüber hinaus zwei altenpflegespezifische Formen, in denen die Zeit zur individuellen Reproduktion durch betriebliche Anforderungen beschnitten wird. Erstens ist es für die Befragten problematisch, dass sie in ständig wechselnden Schichten arbeiten, auf deren monatliche Festlegung sie kaum Einfluss haben. Uns wird mehrfach berichtet, dass weder auf regelmäßige noch auf einmalige private Termine von Seiten der Dienstplanung Rücksicht genommen wird; lediglich durch individuelles Tauschen werden entsprechende Räume eröffnet. Zweitens gibt es die verbreitete Praxis von spontaner Schichtübernahme in der dienstfreien Zeit, wodurch regelmäßige Freizeitaktivitäten und die Begegnung mit Freunden oft unplanbar oder unmöglich werden. Im Vergleich hierzu wird bei den als Facharbeitern und Facharbeiterinnen Beschäftigten aus der Metallbranche deutlich, wie sehr - trotz einer wahrgenommenen sukzessiven Verschlechterung tariflicher Regelungen durch Betriebsvereinbarungen - hier kollektive Vereinbarungen, insbesondere die klar definierten Schichtregelungen, wirksam sind. Auch ausgedehnte Arbeitszeiten bieten so ein gewisses Maß an Planungssicherheit. Kollektive Regelungen ermöglichen den Facharbeitern einerseits eine klare Grenzziehung gegenüber Erwartungen des Unternehmens. Andererseits haben sich mehrere von ihnen für eine zeitintensive berufliche Weiterbildung außerhalb der betrieblichen Arbeitszeit entschieden, um ihre zukünftigen beruflichen Chancen zu verbessern, sodass auch sie unter Zeitmangel für Paar-, Familien- und Sozialleben leiden. Eine weitere Form der Entgrenzung erfahren ferner die Ingenieurinnen und Ingenieure. Bei ihnen ufert die eigentlich als Normalarbeitstag angelegte Arbeitszeit regelmäßig aus, weil auch morgens früh oder abends Präsenz im Betrieb erwartet wird oder notwendig ist.

(4) Zu dieser Mehrebenenanalyse vgl. Winker/Degele 2009. 
Diese Unsicherheit in der alltäglichen Lebensgestaltung ist keinesfalls gleichzusetzen mit der Prekarität, in der die Pflegekräfte leben. Die Ingenieurinnen und Ingenieure haben beispielsweise finanzielle Ressourcen, um die Versorgung ihrer Kinder qualitativ hochwertig zu gestalten. Dennoch denken wir, dass die Brüchigkeit ihres Lebenszusammenhangs, die in unseren Interviews deutlich wird, ernst genommen werden sollte, wenn ausgelotet wird, unter welchen Bedingungen sich Beschäftigte für eine Veränderung ihrer Lebensbedingungen einsetzen (könnten). Ein relativ reibungsloser Alltag setzt ein rigides Management menschlicher Bedürfnisse voraus - sowohl der eigenen als auch der von Familienangehörigen. Diese Lebensweise findet bei den Befragten zwar weitgehende Zustimmung, wird aber dennoch aufgrund der dafür nötigen Strenge sich selbst und anderen gegenüber als anstrengend empfunden.

Eine Grenze dieser durchflexibilisierten und -organisierten Lebensweisen ist, dass für fast alle Befragten eine ausreichende Selbstsorge prekär wird und zwar auch bei Personen, die keine oder geringe Sorgeverantwortung für andere Menschen tragen. Zeit für Reproduktion und Muße wird ständig infrage gestellt, dadurch drohen absehbar psychische und physische Beeinträchtigungen. Als Ursachen dafür benennen Interviewte aus allen drei Bereichen vor allem betriebliche Anforderungen. Neben der eingeschränkten Sorge um sich selbst treten dadurch Zeitkonflikte auf, die die Herstellung familiärer Gemeinsamkeit bzw. freundschaftlicher Beziehungen brüchig werden lassen.

In vielen Interviews sehen wir eine ständige „innere Zerrissenheit" (Jurczyk et al. 2009, S. 212ff.), die sich in Form von Zeitnöten und emotionalen Spannungen äußert, beispielsweise dann, wenn betriebliche Anforderungen eine Familiengründung gänzlich verhindern oder bereits Familienbeziehungen zerstört haben. Bei den (wenigen), die ein reibungslos gelingendes Familienleben schildern, sind die Kinder entweder schon erwachsen und die Sorgearbeit ist dadurch gering. Oder sie stellen die Befriedigung eigener Bedürfnisse zugunsten der Familie und Kinder zurück, um den Anforderungen aus Erwerbs- und Sorgearbeit nachkommen zu können.

\subsection{Subjektive Verarbeitungsweisen entgrenzter Verhältnisse}

Obschon wir in fast allen Interviews Hinweise auf die beschriebenen Belastungen finden, die mit den entgrenzten Erwerbsarbeitsverhältnissen einhergehen, fällt zugleich auf, dass es ganz verschiedene Formen gibt, mit denen unsere Interviewpartnerinnen und -partner den steigenden Druck verarbeiten. Wir haben die Interviews anhand folgender Fragen in Gruppen eingeordnet: Wie beschreiben sie Belastungen und Anforderungen? Was sind für sie wesentliche Konflikte? Welches Handeln ist ihrer Ansicht nach deshalb begründet? Dabei ließen wir die gesellschaftliche Positionierung (Erwerbsarbeitsfeld, familiäre Sorgeverpflichtungen) zunächst unberücksichtigt. Auf diese Weise vermieden wir, aufgrund von Vorannahmen über die jeweils dominanten Probleme in den unterschiedlichen Feldern Übereinstimmungen über die Berufsgrenzen hinweg aus dem Blick zu verlieren oder vorschnelle Vergleiche anzustellen. Insgesamt schälten sich vier unterschiedliche Verarbeitungsweisen von Belastungen heraus, die wir im Folgenden vorstellen. Wie wir zum Schluss kurz aufzeigen, wurde bei der Diskussion der Ergebnisse klar, dass die verschiedenen Ausprägungen der Subjektkonstruktionen zugleich auch über die Unterschiede zwischen den Gruppen hinweg stark mit den unterschiedlichen Anforderungen, aber auch Arbeits- und Lebenskulturen zusammenhängen, die in den drei Berufsfeldern Pflege, industrielle Facharbeit und Ingenieurwesen existieren.

\subsubsection{Zufriedene Einpassung trotz Belastungen}

Die erste Gruppe von Befragten beschreibt sich als zufrieden und ihre Erwerbsarbeitsbedingungen als wichtige Voraussetzung für diese Zufriedenheit, die eine gelungene Kombination der verschiedenen Lebensbereiche einschließt. In dieser Gruppe gibt es ausschließlich männliche Ingenieure und weibliche Pflegekräfte. Die Subjektkonstruktionen schließen in ihrer Unterschiedlichkeit in mehreren Hinsichten an traditionelle Geschlechterverhältnisse und Berufskulturen an.

In den Interviews mit den drei Frauen aus der Pflege wird deutlich, dass ihre Erwerbsarbeitszeiten stark entgrenzt sind, und es gibt Hinweise auf hohe Arbeitsanforderungen, gesundheitliche Beeinträchtigungen und zahlreiche Überstunden. Allerdings erwähnen sie dies eher nebenbei, ohne dass es ihre Zufriedenheit beeinträchtigt. Erwerbsarbeit und darin entfaltete Sorge für andere ist für sie wesentlicher Ausdruck ihrer Persönlichkeit. Wichtig ist ihnen die Anerkennung als Mensch. Sie äußern in den Interviews keinen Wunsch nach Abgrenzung gegenüber betrieblichen Anforderungen. Ihre Handlungsmöglichkeiten sehen sie vielmehr darin, sich aktiv einzubringen, damit der Arbeitsprozess möglichst gut läuft. So berichtet eine Altenpflegerin, dass sie ihr Leben nur von Woche zu Woche plant, um einerseits den häufigen Anfragen des Heimes nach spontanen Diensten nachkommen zu können, um andererseits aber auch Raum für Erholungszeiten von der erschöpfenden Pflegearbeit zu wahren. Obwohl sie auf vieles im Leben verzichtet, erläutert sie zugleich, dass ihr gesamtes Umfeld dies akzeptieren müsse. Sie erwartet, „dass mein Freundeskreis das versteht und darauf Rücksicht nimmt. Im Laufe der Jahre ist es eben halt so raus gekommen, dass die Menschen, die ich jetzt um mich habe, es mir wert sind, oder ich es ihnen wert bin, dass sie auch darauf verzichten.“

Ähnlich beschreiben es auch die anderen Frauen dieser Gruppe: Die Ausrichtung des gesamten Lebenszusammenhangs an den betrieblichen Anforderungen wird einerseits durch die entsprechende Akzeptanz vonseiten der Familie und Freunde ermöglicht, andererseits durch die eigene Fähigkeit, Wünsche anderer ggf. auch zurückzuweisen. Sie 
beschreiben ihre Familien als Unterstützung, insofern diese ihre häufige Abwesenheit im Alltag akzeptieren. Die familiäre Sorgearbeit lassen alle drei allmählich hinter sich, da die Kinder fast erwachsen sind. Auf dieser Basis können sie heute als Lohnabhängige Sorge für andere übernehmen. Für sie selbst ist das Lebensqualität, zumindest wird es in den Interviews so artikuliert.

Die männlichen Ingenieure dieser Gruppe sind die Einzigen in unserem Sample, die keine Prekarisierungstendenzen thematisieren. Sie ähneln den Pflegekräften in der Äußerung großer Zufriedenheit, weichen in ihren Selbstbildern aber stark von ihnen ab. Sie bewerten ihre Erwerbsarbeit durchaus ähnlich als inhaltlich interessant, aber auch als Mittel zum Zweck, der in anderen Lebensbereichen liegt. Diese Sichtweise geht einher mit einem Selbstbild von klarer Grenzziehung gegenüber der Erwerbsarbeit. Zwei sind kinderlos, einer ist alleinerziehender Vater und teilzeiterwerbstätig. Familienleben verstehen alle drei Männer als Verwirklichung von Lebensqualität. Der Vater bezieht dies auf sein aktuelles Handeln, die anderen beiden auf ihren Zukunftswunsch nach Kindern. Dass Letzterer sie hindern könnte, ihre Erwerbsarbeit weiterhin mit anderen Interessen in Einklang zu bringen, erwägen sie nicht.

\subsubsection{Verwirklichung eigener Lebensentwürfe im Konflikt mit Belastungen}

Die Befragten dieser Gruppe schildern im Unterschied zur ersten Gruppe zwar Belastungen, die sich daraus ergeben, dass sie nahezu ständig verfügbar und leistungsbereit sein müssen. Ihre Subjektkonstruktionen gleichen jenen der ersten Gruppe aber darin, dass sie sich selbst als gestaltungsmächtig und zufrieden beschreiben. Ihre Lebensweisen, mit denen sie sich den hohen Anforderungen anpassen, konstruieren sie als Ausdruck ihrer eigenen Persönlichkeit und Prioritätensetzung. Sie schildern zwar Einschränkungen ihrer Lebensqualität, aber diese sehen sie als notwendigen Kompromiss, um selbstgesteckte Ideale oder Ziele zu verwirklichen. Kollektive Veränderungspraxen erscheinen nicht nötig, da ihnen eine der eigenen Persönlichkeit entsprechende Lebensführung gelingt. Dabei ist für einige der Befragten dieser Gruppe ihre Erwerbstätigkeit der zentrale Aspekt ihrer Subjektkonstruktionen; andere hingegen stellen sich als Familienmenschen dar. Diese unterschiedlichen Subjektkonstruktionen korrespondieren mit dem übernommenen Maß an Sorgeverantwortung.

Fünf Personen dieser Gruppe (drei Ingenieurinnen, eine Facharbeiterin und ein Facharbeiter) tragen geringe oder keine Sorgeverpflichtung. Für Erstere baut die Konstruktion einer gelingenden Verwirklichung der eigenen Lebensweise darauf auf, dass es ihrer Identität als „Arbeitstier und Workaholic" entspricht, sich vollständig in die Anforderungen der Erwerbsarbeit einzufügen. Sie präsentieren sich als Menschen, die dafür aus Überzeugung Familie oder Partner dauerhaft einem strikten Management unterwerfen. So sieht sich eine Abteilungsleiterin, die mit ihrem Mann und ihren zwei Kindern zusammenlebt, zuhause in einer klassischen „Vaterrolle“, für die sie sich bewusst entschieden hat. Nur am Wochenende und im Urlaub gibt es Zeit für das Familienleben. Sie beschreibt ihren Partner und sich als „Team“, das seinen Familienalltag regelt und einvernehmlich darauf basiert, Bedürfnissen nach Paarleben nur in den dafür vorgesehenen Zeiten nachzugehen. Die Facharbeiterin und der Facharbeiter erleben es stärker als Mangel, dass die Erwerbsarbeit und eine darauf bezogene Weiterbildung, mit der sie langfristig Freiheitsgewinne zu erreichen hoffen, aktuell keinen Raum für ein soziales Leben lassen. Allerdings sehen sie dies als eine vorübergehende Phase an.

Die anderen drei Befragten haben ein hohes Maß an Sorgearbeit; sie sehen sich in Übereinstimmung hiermit primär als Familienmenschen. Hier handelt es sich um zwei Frauen, die in der Pflege arbeiten, und einen Facharbeiter. Die beiden Frauen konstruieren sich als grundsätzlich zufrieden mit ihrer Mutterrolle. Um dies mit entgrenzter Erwerbsarbeit zu vereinbaren, verzichten beide explizit darauf, ihren individuellen Interessen nachzugehen. Eine von ihnen erzählt, dass ihr neben der Erwerbsarbeitszeit von 30 Wochenstunden und einer Familie mit Mann und zwei Kindern kaum Zeit alleine bleibe. Neben dem Ausführen des Hundes sei „der Rest des Tages eigentlich nur Familie und die Arbeit. Also ich richte eigentlich mein Leben nach deren Leben und Wünschen. “ Dies befürwortet sie, da „eine Frau wirklich der ganze Kopf der Familie“ sei. Der Facharbeiter sieht sich zwar ebenfalls als jemand, für den die aktive Verantwortung als Vater zentral ist, kann aber daneben ein Fernstudium und Engagement in der Elternarbeit realisieren. Während die beiden Frauen aus der Pflege individuell dafür sorgen, dass sie neben der Arbeit im Pflegeheim die familiäre Sorgearbeit schaffen, erhält er für diese Aktivitäten Unterstützung: Sein Team im Betrieb nimmt bei Schichtregelungen Rücksicht auf seine Termine; seine Partnerin übernimmt nachmittags die Kinderbetreuung.

\subsubsection{Unlösbare Konflikte zwischen Grenzziehung und Ohnmacht}

Die Befragten dieser dritten Gruppe beschreiben deutlich, dass sie unter den hohen alltäglichen Belastungen leiden. Sie schildern, dass Selbstsorge und eigene Interessen zu kurz kommen und sehen dies auch bei Kolleginnen und Kollegen. Ihre Subjektkonstruktionen prägt der Widerspruch zwischen einem hohen persönlichen Interesse an den Inhalten der Erwerbsarbeit und den belastenden Bedingungen, unter denen sie diese gestalten. Dieser Widerspruch wird von ihnen nicht benannt oder bewusst bearbeitet. Stattdessen versuchen sie kontinuierlich, individuell gegenüber belastenden Situationen Grenzen zu ziehen, sind aber zugleich gegenüber den Verhältnissen, in denen sie tätig sind, relativ ohnmächtig. Dabei führen Konflikte zu einer permanenten Unzufriedenheit.

Ein Beispiel dafür ist die Wahrnehmung einer Ingenieurin und Teamleiterin, die erklärt, dass weder sie 
noch ihre Kolleginnen bzw. Kollegen eine funktionierende Strategie hätten, die flexible Arbeitszeitgestaltung in ihrem eigenen Interesse zu nutzen. Sie sieht dies in einer Haltung zur Erwerbsarbeit begründet, in der die Einschränkung eigener Bedürfnisse für ein gutes Arbeitsergebnis in Kauf genommen wird. Sie setzt damit die hohen betrieblichen Anforderungen mit den eigenen Ansprüchen an gute Arbeit gleich, die sie auch unter schwierigen Bedingungen nicht aufgeben möchte. In der Folge setzt sie gegenüber dem Unternehmen selten Grenzen. Gleichzeitig leidet sie darunter, dass ihr Leben so stark von der Erwerbsarbeit bestimmt wird. Sie beschreibt es als „langweilig“ und berichtet, dass sie oft „müde und angenervt" nach Hause komme und ihr die Energie fehle, abends zu lesen oder kreativ zu sein. Ihr Ehe- und Familienleben erlebt sie - vor dem Hintergrund, dass ihr Sohn fast erwachsen ist - als Ort der Regeneration. Zuhause dürfe sie mit all ihrer Erschöpfung „einfach sein“ und müsse keine Ansprüche erfüllen.

Bei den Befragten, bei denen im Unterschied hierzu ein höherer Bedarf nach Zeit und Kraft für familiäre Sorgeverpflichtungen existiert, ist dies durchweg das zentrale Konfliktfeld. Ein Pflegehelfer erzählt, dass bei der Dienstplanung keine Rücksicht auf Zeit für seinen zehnjährigen Sohn genommen wird. Eine Ingenieurin und eine Pflegerin teilen die Erfahrung, dass die Erwerbsarbeit keinen Raum lässt, überhaupt ein Familienleben aufzubauen. Während die Ingenieurin es aufgrund ihrer langen Arbeitszeiten als unmöglich beschreibt, einen Partner zu finden, bringt die Pflegerin ihr Leben folgendermaßen auf den Punkt: „Geschieden, alleine lebend, eine Tochter, die ich seit zwei, mindestens drei Monaten nicht gesehen habe. Eine Schwester, die ein paar Türen weiter wohnt, die ich auch sehr sporadisch sehe. Aus dem einfachen Grund, weil die Arbeitszeiten scheiße sind."

Auffallend ist ein Unterschied in der Konstruktion der eigenen Handlungsfähigkeit: Die zwei fest angestellten Ingenieurinnen konstruieren sich in der Form als handlungsmächtig, dass sie mittelfristig durch den Wechsel des Arbeitgebers bessere Lebensbedingungen erreichen können. Dieses Selbstbild steht dabei im Gegensatz dazu, dass bei beiden Erschöpfung und ungelöste Zeitprobleme seit Langem ihren Alltag prägen und sie sich dennoch bisher kein besseres Unternehmen gesucht haben. Ein Ingenieur in Leiharbeit und zwei Pflegerinnen erläutern hingegen, dass sie Anforderungen vonseiten des Arbeitgebers nicht zurückweisen können. Der Ingenieur begründet dies mit seinem prekären Beschäftigungsstatus, die Pflegekräfte sehen Überstunden, Schichtdienst und spontane Dienste hingegen als unveränderbaren Bestandteil der Altenpflegearbeit. Für ihre Subjektkonstruktion ist es zentral, dass sie das Wohl der Bewohner aufrechterhalten, indem sie im Erwerbsarbeitsalltag - egal in welchem Betrieb - leistungsbereit sind.

\subsubsection{Politisches Engagement im Betrieb}

Schließlich gab es in unserem Sample Befragte, die als Reaktion auf den Leistungsdruck gewerkschaftlich oder als Betriebsräte aktiv sind oder sich positiv auf solche institutionalisierten Handlungsformen beziehen. Obschon auch bei ihnen teilweise Begeisterung für den Beruf zum Ausdruck kommt, sind die Interviews stark vom Leiden unter den betrieblichen Arbeitsbedingungen, ersten psychosomatischen Stresssymptomen und Angst vor weiteren Erkrankungen geprägt. Gemeinsam ist ihnen, dass ihr Kollegenkreis sich kaum für kollektives Handeln interessiert und sie daher (teilweise erfolglos) viel Überzeugungsarbeit leisten. Gemeinsam ist ihnen auch, dass sich ihr politisches Handeln nur auf den Betrieb bezieht. Wie die familiäre Verantwortung mit den Anforderungen aus der Erwerbsarbeit und der politischen Aktivität verbunden werden kann, hierfür finden alle Interviewten rein private Lösungen. Familienleben ist klassisch im engen Kreis von Ehepartnern und Kindern bzw. Eltern organisiert, weitere Personen oder Netzwerke spielen kaum eine Rolle.

Fragt man nach den Ausgangspunkten für die Entscheidungen der Befragten, über rein individuelle Bewältigungsstrategien hinauszugehen, so finden sich tendenziell drei Antworten: Zwei Befragte werden aus einem grundsätzlich kritischen Gesellschaftsbild heraus aktiv und sehen es als notwendig an, diese Kritik in Form konkreter betrieblicher Arbeit praktisch umzusetzen. Zwei andere (beide sind Facharbeiter) beschreiben, dass Gewerkschaft mit Beginn ihrer Tätigkeit im Unternehmen ein zentraler sozialer Kontext für sie wurde, deren Grundverständnis von sozialen Rechten (und Pflichten) heute ihre Identität prägen. Bei zwei Pflegerinnen wird deutlich, dass die Entscheidung, im Betrieb aktiv zu werden, von der Unterstützung im persönlichen Umfeld abhängt. Da diese oft nicht von Kollegen kommt, sind hier andere politisch Aktive nötig, die sie ermutigen oder gemeinsam mit ihnen handeln.

Bei den Berichten darüber, inwiefern dieses kollektive Engagement auch dabei nützt, im Alltag gegenüber dem Unternehmen eine Grenze zu ziehen und sich persönlichen Freiraum zu verschaffen, fällt auf, wie unterschiedlich die Handlungsräume in den Berufsfeldern sind. Dabei korrespondieren unterschiedliche Strukturen mit den jeweiligen subjektiven Verortungen gegenüber Unternehmen bzw. Vorgesetzten. So bezieht sich ein Mechaniker auf Arbeitsrechte und Tarifvertrag, an deren Grenzen er sich auch exakt hält, wenn er sein „Recht auf Freizeit“ und seine Zeit für politische Arbeit gegenüber Kollegen und Vorgesetzten verteidigt. Im Unterschied hierzu bestehen zwar auch zwei Pflegerinnen im Interview darauf, wie wichtig es sei, die eigene Zeitplanung nicht für betriebliche Belange und Anforderungen zu opfern. Dies beinhaltet allerdings, dass sie dennoch für kurzfristige Anfragen nach Zusatzschichten generell offen sind, weil sie deren Notwendigkeit sehen. D.h. die grundsätzliche Priorisierung persönlicher Zeitbedarfe geschieht hier stets in einem Rahmen, in dem ein akuter Personalbedarf des Heims mitgedacht wird. 


\section{Unterschiedliche Ansatzpunkte für kollektives Handeln}

Unsere Interviews bestätigen, dass Belastungen von den Betroffenen überwiegend individuell bewältigt und kompensiert werden - selbst dann, wenn sie als eine mit Kolleginnen und Kollegen geteilte Erfahrung beschrieben werden. Auf die Entgrenzung von Anforderungen wird in der Regel mit der Begrenzung der eigenen Bedürfnisse reagiert, was teils als Ergebnis von Zwängen wahrgenommen wird, teils als Ausdruck der eigenen Persönlichkeit; bisweilen mischen sich beide Perspektiven. Eine wesentliche Form, mit der innerhalb solcher Selbstbegrenzungen dennoch Zufriedenheit hergestellt wird, ist die hohe Identifikation mit der Erwerbsarbeit. So können wir für einige Altenpflegekräfte (besonders in der ersten Gruppe, vgl. 3.2.1) zwar kritisch feststellen, dass sie ihren gesamten Le benszusammenhang den Flexibilitätsansprüchen der Pflegeheime unterordnen. In ihren subjektiven Wahrnehmungsweisen, wie sie in den Interviews deutlich werden, erleben sie selbst dies allerdings als Ermöglichungsstruktur, die es ihnen erlaubt, aktiv zum Gelingen der Pflegearbeit beizutragen. Ähnliches passiert (besonders in der zweiten Gruppe, vgl. 3.2.2), wenn für einige Ingenieurinnen - trotz der belastenden Bedingungen - ihre Identität als „Workaholic“ den Ausgangspunkt dafür bildet, dass sie ihren Lebenszusammenhang als gelungen beschreiben und das rigide Management von Bedürfnissen als Selbstverständlichkeit hinnehmen. Für einige Frauen, die in der Pflege arbeiten, ist die Identifikation als Familienfrau und Mutter die Begründung, auf eigene Interessen neben Erwerbs- und Sorgearbeit zu verzichten.

(Potenzielle) Brüche in diesen Konstruktionen von befriedigenden Lebensentwürfen fanden wir in unterschiedlicher Form: So bedauern die Ingenieurinnen, dass ihnen Leichtigkeit und Unbeschwertheit fehlen, dass sie einen hohen Lebensstandard und den Wunsch nach mehr Freizeit für unvereinbar halten und einen Großteil ihres Einkommens dafür aufwenden, um ,das Thema Familie und diese ganze Organisation der Arbeit wiederum organisiert zu kriegen“. Dabei ist der vermeintliche Ausweg, das Unternehmen zu wechseln, unseres Erachtens insofern eine Illusion, weil sich vermarktlichte Strukturen weitgehend durchgesetzt, also verallgemeinert haben. Die Pflegekräfte kamen dann ins Zweifeln, wenn sie beispielsweise feststellten, dass sie aufgrund der Zentralisierung der familiären Sorgearbeit „in ihrer eigenen Planung selbst gar nicht vorkommen“. Andere berichten trotz aller Akzeptanz, die ihre Familie der entgrenzten Erwerbsarbeit entgegenbringt, von „schlechtem Gewissen“, wenn sie Bedürfnisse der Familie zurückweisen. Mehrfach äußern sie auch Wünsche nach Weiterbildung und beruflicher Veränderung, deren Verwirklichung sie gleichfalls als unmöglich beschreiben. Das Prekäre an der Zufriedenheit der Pflegekräfte wird darüber hinaus vor allem daran deutlich, dass in all ihren Interviews Hinweise auf gesundheitliche Einschränkungen zu finden sind - wenn auch oft nur als Randbemerkung, was umso mehr deren große Normalität verdeutlicht

Auch dort, wo die Konflikte im Alltag als sehr beeinträchtigend beschrieben werden (Gruppe drei, vgl. 3.2.3), versuchen Menschen nicht zwangsläufig, ihre Lebensbedingungen zu verändern. Auffallend ist, dass viele sich selbst auf die direkte Nachfrage hin als Menschen beschreiben, die gut Grenzen ziehen können, während ihre Alltagsbeschreibungen vor allem verdeutlichen, wie sie darin scheitern, da sie ständig überlastet sind und dies ihre Lebensqualität einschränkt. Andere sehen keine anderen Handlungsmöglichkeiten, als sich mit den Gegebenheiten abzufinden und möglichst gut „durchzukommen“.
Wenn wir im kontrastierenden Vergleich mit den Interviewpersonen in Gruppe vier (vgl. 3.2.4) fragen, wie es dazu kommt, dass Menschen sich eben doch für Veränderungen einsetzen, so können wir feststellen: Was es in jedem Fall braucht, ist der persönliche Kontakt und Austausch mit Menschen, die die grundsätzliche Veränderbarkeit der belastenden Bedingungen für denkbar halten und hierzu praktische Wege aufzeigen. Was allerdings offen bleibt und unseres Erachtens weiterer Forschung bedarf, ist die Frage, wann Menschen offen dafür sind, die Belastungen, deren Bewältigung für sie zur Normalität geworden ist, als „Problem“ zu thematisieren, dessen Lösung oder Bearbeitung ihnen auf Dauer nicht mehr alleine gelingt, sondern das eines gesellschaftsbezogenen Handelns bedarf. Insgesamt erlebten wir in den Interviews häufig, dass die Begriffe „Problem“, „Konflikt“ und „Widersetzung“ negativ besetzt sind und dass sich unsere Gesprächspartner hiervon distanziert.

Beschäftigte in Ingenieursberufen - sei es in Festanstellung oder in Leiharbeit -, Facharbeiterinnen und Facharbeiter sowie Altenpflegekräfte sind sehr unterschiedlichen Entgrenzungsprozessen ausgesetzt, die sich nicht gleichsetzen lassen, da sowohl die materiellen Ressourcen als auch die Arbeitsbedingungen grundlegend verschieden sind. Dennoch lohnt es sich unseres Erachtens in allen drei Bereichen, die Fragilität der Lebenszusammenhänge und die fehlende Befriedigung im Lebenskontext (in ihrer Unterschiedlichkeit) als Probleme ernst zu nehmen. Hier können Gespräche ansetzen, die den Beschäftigten die Möglichkeit geben, die Normalisierung der hohen Belastungen und ihren eigenen Umgang damit zu überdenken. Betrieblich Aktive können Reflexionsprozesse darüber anstoßen, ob die Bedingungen, die diese Belastungen hervorbringen, tatsächlich unveränderbar sind und welcher Zusammenhang zwischen Alltagserfahrungen und politischen Strategien besteht. In der Altenpflege braucht es perspektivisch eine Kultur der Grenzziehung, die von Vorgesetzten respektiert wird und deren strukturelle Grundlagen politisch gestärkt werden, die aber auch von Beschäftigten - ggf. im Konflikt - entwickelt werden muss. Unter den Befragten aus dem Industrieunternehmen unseres Samples ist Grenzziehung zumindest als Ideal verbreiteter. Hier gilt es zu thematisieren, inwiefern dies im Zuge von Unternehmensumstrukturierungen nicht mehr nur durch individuelles Handeln in die Praxis umzusetzen ist und wo Grenzziehungen Selbstbeschränkung bedeuten, statt den neuen Leistungspolitiken Grenzen zu setzen.

\section{LITERATUR}

Bispinck, R. (2003): Das deutsche Tarifsystem in Zeiten der Krise. Streit um Flächentarif, Differenzierung und Mindeststandards, in: WSI-Mitteilungen 56 (7), S. 395-404, http://www.boeckler.de/wsi_24228_24238.htm

Boes, A./Kämpf, T. (2010): Zeitenwende im Büro: Angestelltenarbeit im Sog der Globalisierung, in: WSI-Mitteilungen 63 (12), S. 611-617, http://www.boeckler. de/wsi_25505_25514.htm

Böhle, F. (1999): Nicht nur mehr Qualität, sondern auch höhere Effizienz - subjektivierendes Arbeitshandeln in der Altenpflege, in: Zeitschrift für Arbeitswissenschaft 53 (3), S. 174-181

Dörre, K. (2002): Kampf um Beteiligung. Arbeit, partizipatives Management und die Gewerkschaften, in: Kurswechsel 17 (2), S. 64-76

Dunkel, W. (2005): Zur Lebensführung von Pflegekräften, in: Schroeter, K. R./ Rosenthal, T. (Hrsg.): Soziologie der Pflege. Grundlagen, Wissensbestände und Perspektiven, Weinheim, S. 227-246 
Glißmann, W. (2000): Die neue Selbstständigkeit in der Arbeit. Maßlosigkeit und Ökonomisierung der „Ressource Ich“, in: J. Peters (Hrsg.): Dienstleistungsarbeit in der Industrie. Ein gewerkschaftliches Gestaltungsfeld, Hamburg, S. $50-80$

Holzkamp, K. (1983): Grundlegung der Psychologie, Frankfurt a.M. Jurczyk, K./Schier, M./Szymenderski, P./Lange, A./Voß, G. G. (2009): Entgrenzte Arbeit - entgrenzte Familie. Grenzmanagement im Alltag als neue Herausforderung, Berlin

Jürgens, K. (2006): Arbeits- und Lebenskraft. Reproduktion als eigensinnige Grenzziehung, Wiesbaden

Jürgens, K. (2011): Prekäres Leben, in: WSI-Mitteilungen 64 (8), S. 379-385, http://www.boeckler.de/wsi_37524_37536.htm

Klenner, C./Pfahl, S. (2011): Prekarisierung im Lebenszusammenhang - Bewegung in den Geschlechterarrangements?, in: WSI-Mitteilungen 64 (8),

S. 416-422, http://www.boeckler.de/wsi_37524_37552.htm

Kratzer, N. (2003): Arbeitskraft in Entgrenzung. Grenzenlose Anforderungen, erweiterte Spielräume, begrenzte Ressourcen, Berlin

Kumbruck, C. (2009): Diakonische Pflege im Wandel. Nächstenliebe unter Zeitdruck, Münster

Lehndorff, S. (2003): Marktsteuerung von Dienstleistungsarbeit, in: Dörre, K./ Röttger, B. (Hrsg.): Das neue Marktregime. Konturen eines nachfordistischen Produktionsmodells, Hamburg, S. 153-171

Lehndorff, S. (2005): Arbeitszeitregulierung unter Druck. Über die schwierige Suche nach einem neuen Flexibilitätskompromiss, in: Kronauer, M./Linne, G. (Hrsg.): Flexicurity. Die Suche nach Sicherheit in der Flexibilität, Berlin,

S. $205-220$

Lessenich, S. (2008): Die Neuerfindung des Sozialen. Der Sozialstaat im flexiblen Kapitalismus, Bielefeld

Lohr, K./Nickel, H. M. (2005): Subjektivierung von Arbeit - Riskante Chancen. Das Paradoxon von erweiterter Teilhabe und sozialer Verwundbarkeit - Problemaufriss, in: Dies. (Hrsg.): Subjektivierung von Arbeit. Riskante Chancen, Münster, S. 207-239

Markard, M. (2011): Handlungsfähigkeit und subjektive Funktionalität oder: ein Plädoyer gegen psychologischen Determinismus, in: Wiebel, B./ Pilenko, A./ Nintemann, G. (Hrsg.): Mechanismen psychosozialer Zerstörung. Neoliberales Herrschaftsdenken, Stressfaktoren der Prekarität, Widerstand, Hamburg,

S. $19-32$

Matuschek, I./Kleemann, F./Voß, G. G. (2008): Subjektivierte Taylorisierung als Beherrschung der Arbeitsperson, in: Prokla 38 (1), S. 49-64

Nowak, I. (2007): Von mutigen Männern und erfolgreichen Frauen. Work-LifeBalance in prekarisierten Verhältnissen, in: Kaindl, C. (Hrsg.): Subjekte im Neoliberalismus. Kritische Wissenschaften, Bd. 2, Marburg, S. 59-74

Nowak, I. (2011): Fürsorgliche Praxis als prekäre Lohnarbeit. Fragen an die Erfahrungen der Beschäftigten, in: Das Argument 53 (292), S. 381-391

Nowak, I./Hausotter, J./Winker, G. (2012): Handlungsfähigkeit in entgrenzten Verhältnissen. Subjektkonstruktionen von Beschäftigten in Industrie und Altenpflege, Hamburg, Projektbericht online verfügbar unter http://doku.b.tuharburg.de/volltexte/2012/1138/

Peters, K./Sauer, D. (2005): Indirekte Steuerung - eine neue Herrschaftsform Zur revolutionären Qualität des gegenwärtigen Umbruchprozesses, in: Wagner, H. (Hrsg.): „Rentier' ich mich noch?”, Neue Steuerungskonzepte im Betrieb, Hamburg, S. 23-58
Schweiger, P. (2011): Wir haben zwar Geduld, aber keine Zeit. Eine Ethnografie subjektivierter Alltagsstile in der ökonomisierten Altenpflege, München Voß, G. G./Pongratz, H. J. (1998): Der Arbeitskraftunternehmer. Eine neue Grundform der Ware Arbeitskraft?, in: Kölner Zeitschrift für Soziologie und Sozialpsychologie 50 (1), S. 131-158

Voß, G. G./Wachtler, G./Böhle, F. (Hrsg.) (2010): Handbuch Arbeitssoziologie Wiesbaden

Winker, G./Carstensen, T. (2007): Eigenverantwortung in Beruf und Familie. Vom Arbeitskraftunternehmer zur Arbeitskraftmanagerln, in: Feministische Studien 25 (2), S. 227-288

Winker, G./Degele, N. (2009): Intersektionalität. Zur Analyse sozialer Ungleichheiten, Bielefeld

WSI-Mitteilungen (2011): Schwerpunktheft: Prekarisierung der Arbeit - Prekarisierung im Lebenszusammenhang, 64 (8), http://www.boeckler.de/wsi_37524. htm

\section{AUTORINNEN}

IRIS NOWAK, Diplom-Sozialökonomin, Wissenschaftliche Mitarbeiterin in der Arbeitsgruppe „Arbeit-Gender-Technik“ an derTU Hamburg-Harburg. Arbeitsschwerpunkte: Kollektive und individuelle Handlungsfähigkeit in neoliberalen Verhältnissen, Altenpflege/Sorgearbeit.

Iris.Nowak@tu-harburg.de

Jette hausotter, M. A. Gender Studies, Wissenschaftliche Mitarbeiterin in der Arbeitsgruppe "Arbeit-Gender-Technik" an derTU Hamburg-Harburg. Arbeitsschwerpunkte: Arbeits- und Geschlechterverhältnisse im Neoliberalismus, Hochqualifizierte Arbeit, Intersektionalität.

hausotter@tu-harburg.de

GABRIELE WINKER, Prof. Dr., lehrt Arbeitswissenschaft und Gender Studies an derTU Hamburg-Harburg und ist Leiterin der Arbeitsgruppe "Arbeit-GenderTechnik". Arbeitsschwerpunkte: Arbeits- und Geschlechtersoziologie sowie Intersektionalitätsforschung, http://www.tu-harburg.de/agentec.

winker@tu-harburg.de 